\title{
Genome-wide analysis of Tol2 transposon reintegration in zebrafish Igor Kondrychyn ${ }^{1}$, Marta Garcia-Lecea1 ${ }^{1}$, Alexander Emelyanov ${ }^{1,3}$, Sergey Parinov ${ }^{1,3}$ and Vladimir Korzh*1,2
}

Address: ${ }^{1}$ Cancer and Developmental Cell Biology Division, Institute of Molecular and Cell Biology, Singapore, ${ }^{2}$ Department of Biological Sciences, National University of Singapore, Singapore and ${ }^{3}$ Temasek Life Sciences Laboratory, Singapore

Email: Igor Kondrychyn - ikondrychyn@imcb.a-star.edu.sg; Marta Garcia-Lecea - mglecea@hotmail.com; Alexander Emelyanov - sasha@tll.org.sg; Sergey Parinov - parinovs@yahoo.com.sg; Vladimir Korzh* - vlad@imcb.a-star.edu.sg

* Corresponding author

Published: 8 September 2009

BMC Genomics 2009, 10:418 doi:10.1186/147|-2164-10-418
Received: 17 April 2009

Accepted: 8 September 2009

This article is available from: http://www.biomedcentral.com/I47I-2/64/I0/4I8

(c) 2009 Kondrychyn et al; licensee BioMed Central Ltd.

This is an Open Access article distributed under the terms of the Creative Commons Attribution License (http://creativecommons.org/licenses/by/2.0), which permits unrestricted use, distribution, and reproduction in any medium, provided the original work is properly cited.

\begin{abstract}
Background: Tol2, a member of the hAT family of transposons, has become a useful tool for genetic manipulation of model animals, but information about its interactions with vertebrate genomes is still limited. Furthermore, published reports on Tol2 have mainly been based on random integration of the transposon system after co-injection of a plasmid DNA harboring the transposon and a transposase mRNA. It is important to understand how Tol2 would behave upon activation after integration into the genome.

Results: We performed a large-scale enhancer trap (ET) screen and generated 338 insertions of the Tol2 transposon-based ET cassette into the zebrafish genome. These insertions were generated by remobilizing the transposon from two different donor sites in two transgenic lines. We found that $39 \%$ of Tol2 insertions occurred in transcription units, mostly into introns. Analysis of the transposon target sites revealed no strict specificity at the DNA sequence level. However, Tol2 was prone to target AT-rich regions with weak palindromic consensus sequences centered at the insertion site.

Conclusion: Our systematic analysis of sequential remobilizations of the Tol2 transposon from two independent sites within a vertebrate genome has revealed properties such as a tendency to integrate into transcription units and into AT-rich palindrome-like sequences. This information will influence the development of various applications involving DNA transposons and Tol2 in particular.
\end{abstract}

\section{Background}

The transposable element Tol2 from medaka fish is the first functional transposon identified in vertebrates [1]. It belongs to the hAT family (named for hobo, Ac and Tam3) and integrates into host DNA through a "cut-and-paste" mechanism [2]. Recently, a non-autonomous Tol2-based system has been developed as a tool for genome analysis of vertebrates and for highly efficient transgenesis [3-11]. It has been used for both gene trap and enhancer trap (ET) screens [12-14] as well as insertional mutagenesis $[15,16]$. Some of these applications have recently been reviewed $[17,18]$. 
One of the features of non-autonomous transposonbased systems, including Tol2, is that a transposon integrated into a genome can be remobilized if transposase mRNA is available. Previous applications of the transposon system have been based on random integration after co-injection of a plasmid DNA harboring Tol2 and transposase mRNA. Such random integration is attractive for a wide variety of applications ranging from gene discovery to gene therapy. However, the pattern of transposon integration upon remobilization from the donor site can be substantially different from that of plasmid-based integration. For example, the Sleeping Beauty (SB) transposon has a strong tendency to reinsert during in vivo remobilization at loci closely linked to its donor site [19]. Such local hopping could be favorable not only for region-specific mutagenesis [20] but also for region-specific probing of enhancers. However, despite the recent surge of interest in the Tol2 transposon system, its integration and/or reintegration properties have not yet been analyzed in detail.

Using Tol2, we have previously established a collection of stable transgenic zebrafish ET lines and demonstrated that a single copy of a Tol2 transposon-based ET cassette can be remobilized into a new chromosomal location $[13,21]$. Here, we report the results of a genome-wide analysis of Tol2 reintegration in zebrafish initiated from two genomic sites in two different chromosomes.

\section{Results}

\section{Design of the Tol2 transposon remobilization screen}

We used two different ET lines as donors for the remobilization experiments. The first line, SqET33, was established during our pilot enhancer trap (ET) screen [13]. It carries a single insertion of a transposable element in the 3' UTR of a novel gene of the Zic family, zic6 on chromosome 14 (Figure 1A). The second line, SqET33-E20, was established after induced remobilization of a Tol2 transposonbased ET cassette from the SqET33 line [22]. It carries a single insertion located approximately $4.2 \mathrm{~kb}$ upstream of a putative gene $z g c: 66340$ (similar to Axin-1 up-regulated gene 1) on chromosome 24 (Figure 1B). In both lines, stable tissue-specific GFP expression is maintained through at least four generations of breeding, indicating that it is not affected by silencing or epigenetic modification. For both donor lines we confirmed the presence of a single Tol2 transposon insertion by Southern blot hybridization (Figure 1C).

In order to induce remobilization of the Tol2 transposonbased ET cassette from the donor site in SqET33 (referred to as the SqET33 donor site), we initially used mRNA containing only an open-reading frame (ORF) for Tol2 transposase. In this experiment, transgenic fish homozygous for a single Tol2 insertion were outcrossed to wild type fish. The embryos from this cross $\left(\mathrm{F}_{0}\right.$ generation) were injected with transposase mRNA at the one- or two-cell stage. Most of the injected embryos only showed a "donor-type" GFP expression pattern. However, some showed mosaic expression of GFP in somatic cells, mostly in muscle or skin [13]. We did not preselect embryos on the basis of such GFP expression, but used these observations as an indication that a transposition had been triggered. Because we were only interested in heritable Tol2 re-transpositions, the $\mathrm{F}_{0}$ generation was not analyzed in respect of transposon integration into somatic cells. All injected embryos were raised to sexual maturity and crossed to wild type fish, and their progeny $\left(\mathrm{F}_{1}\right.$ generation) were analyzed for changes in GFP expression. We identified injected fish as founders $\left(\mathrm{F}_{0}\right.$ founder) if their progeny showed a new GFP expression pattern that differed from the donor pattern. The $\mathrm{F}_{0}$ founder fish carried the Tol2 reintegrations in the germline. In our screening scheme, only the $F_{1}$ embryos that showed new GFP expression patterns were further analyzed by TAIL-PCR (Figure 1D). Using this strategy, we initially identified 21 $\mathrm{F}_{0}$ founder fish out of 282 injected fish. This corresponded to a $7 \%$ apparent germline transposition rate $\left(1^{\text {st }}\right.$ screen in Table 1). We assumed that the transposase mRNA used at that point was not very effective in Tol2 remobilization. Therefore, in subsequent experiments, we used a modified transposase mRNA containing the 5' and 3' UTRs of the Xenopus $\beta$-globin gene [6]. As a consequence, we identified $103 \mathrm{~F}_{0}$ founder fish out of 268 injected fish in a new round of screening, a much higher apparent germline transposition rate $\left(38 \%, 2^{\text {nd }}\right.$ screen in Table 1$)$.

To test whether the donor site influences transposon remobilization, we used the SqET33-E20 line (referred to as the SqET33-E20 donor site) as a donor. We identified $84 \mathrm{~F}_{0}$ founder fish out of 175 injected fish, with an apparent germline transposition rate similar to that of the $2^{\text {nd }}$ screen $\left(48 \%, 3^{\text {rd }}\right.$ screen in Table 1$)$. Because our screening was based on the appearance of new GFP expression patterns, new insertions that caused no changes in that pattern were not taken into account. Therefore, the actual germline transposition rate for both donor sites should be higher.

In most cases, when $\mathrm{F}_{0}$ fish (heterozygous for $g f p$ ) were outcrossed to wild type fish, GFP-negative and -positive embryos (regardless of their expression patterns) were segregated in an approximately 1:1 ratio. However, we observed altered GFP segregation ratio following remobilization of the Tol2 transposon (see Additional file 1). Out of 725 injected $F_{0}$ fish (from three rounds of screening), 22 produced more than $50 \%$ GFP-positive progeny, suggesting an increase of Tol2 copy number. Thirty-seven injected $\mathrm{F}_{0}$ fish produced less than 50\% GFP-positive progeny, indicating either partial loss of Tol2 or silencing of $g f p$. A similar alteration of GFP segregation has recently been described for re-transposition of the Ds element in zebrafish [23]. Germinal excision without concomitant 

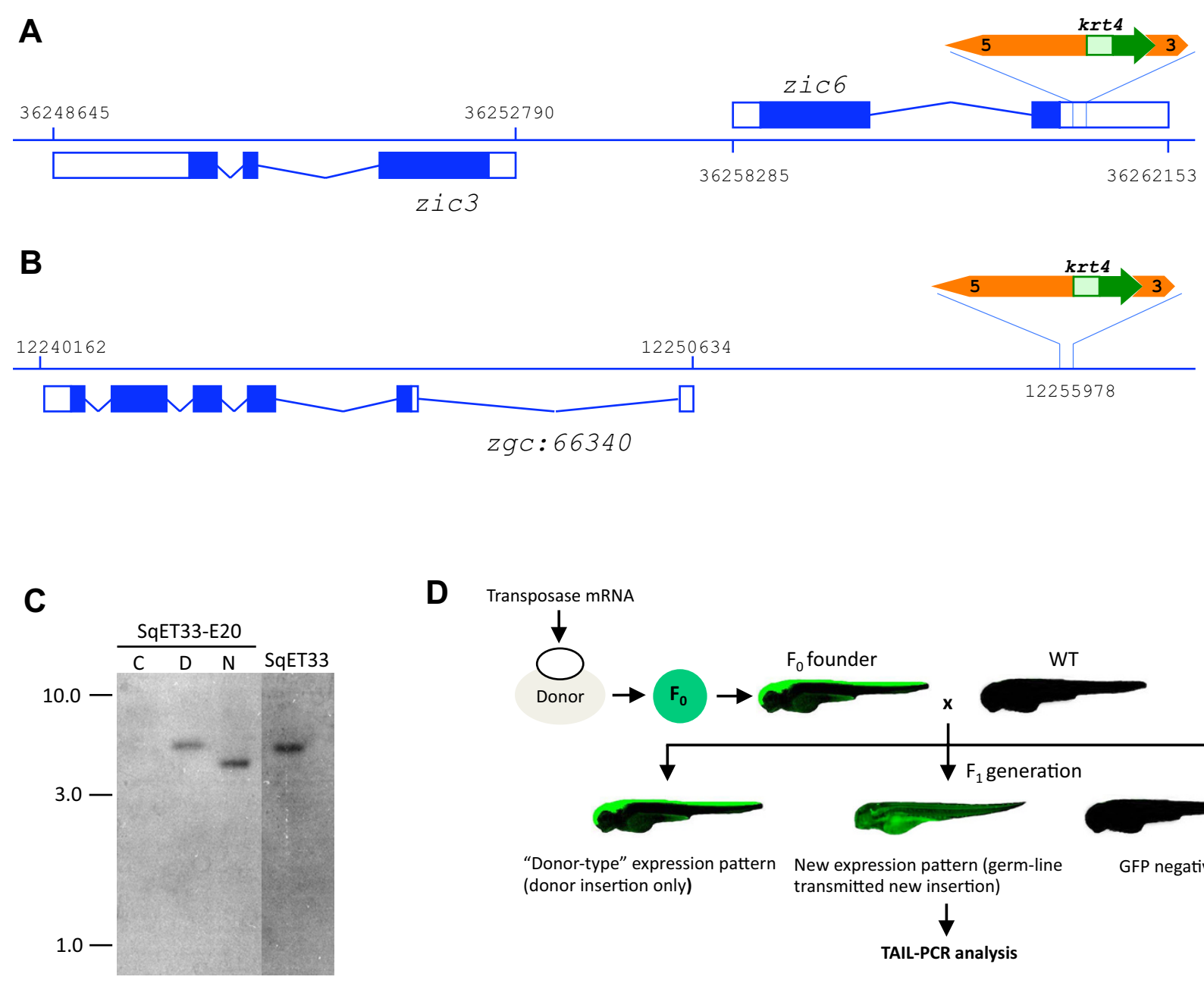

D Transposase mRNA

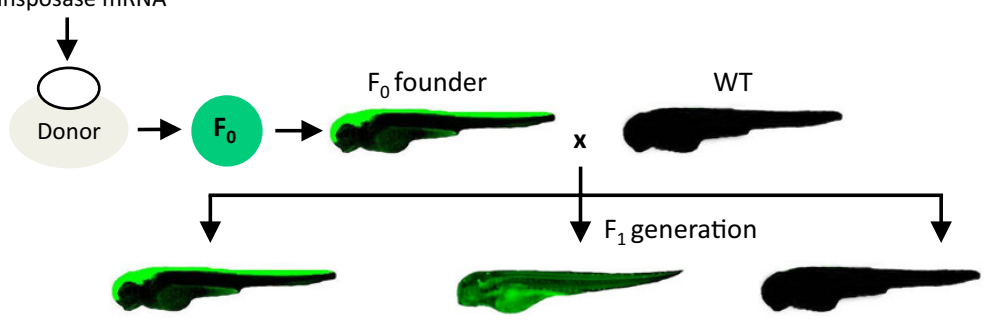

"Donor-type" expression pattern New expression pattern (germ-line (donor insertion only) transmitted new insertion)

GFP negative

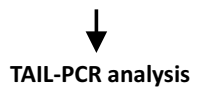

Figure I

Donor sites used for the remobilization screen. A: The SqET33 donor site (Tol2 transposon insertion in the 3' UTR of zic6). B: The SqET33-E20 donor site (Tol2 transposon insertion $4.2 \mathrm{~kb}$ upstream of zgc:66340). The green arrow shows EGFP; krt4 is a minimal promoter; orange arrows represent the $5^{\prime}$ and $3^{\prime}$ ends of Tol2; coding exons are depicted as blue boxes; and UTRs are represented as open boxes. C: SqET33-E20 $F_{0}$ founder fish was outcrossed to wild type and DNA isolated from GFP-negative embryos (negative control, C), GFP-positive embryos showing a SqET33 expression pattern of GFP (donor-type, $\mathrm{D})$, and GFP-positive embryos showing a new expression pattern distinct from the donor one ( $N$ ), were used for Southern blot hybridization. SqET33, DNA from the donor line. D: A scheme of the Tol2 remobilization screen.

transposon reintegration has also been reported for the Ac/Ds transposon in plants [24,25].

Interestingly, about $40 \%$ of $\mathrm{F}_{0}$ founder fish after outcrossing with wild type produced progeny $\left(\mathrm{F}_{1}\right)$ such that individual embryos within a single $\mathrm{F}_{1}$ family showed distinct new GFP expression patterns. The number of these new patterns per single $F_{1}$ family varied from two to seven, suggesting multiple transposon integration events in the germline of a single $\mathrm{F}_{0}$ fish (Table 2 ). In most cases, TAIL-
PCR analysis of individual embryos from such $\mathrm{F}_{1}$ families demonstrated single Tol2 insertions at different positions in the genome. The presence of a single insertion in $F_{1}$ fish suggests that transposition occurred independently in separate germline cells, and Tol2 was transposed by a nonreplicative mechanism, since an $\mathrm{F}_{0}$ fish is heterozygous for a single insert. However, in a few cases, TAIL-PCR detected two or three insertions in one $\mathrm{F}_{1}$ embryo; this was additionally confirmed by Southern blot hybridization (see Additional file 2). 
Table I: Tol2 remobilization screen overview

\begin{tabular}{|c|c|c|c|}
\hline Screen & I sta & 2ndb & $3 r^{c}$ \\
\hline Total $F_{0}$ fish screened & 282 & 268 & 175 \\
\hline Total $F_{0}$ founder fishd & 21 & 103 & 84 \\
\hline Apparent germline transposition rate & $7 \%$ & $38 \%$ & $48 \%$ \\
\hline Number of new insertions ${ }^{f}$ & 23 & 172 & 143 \\
\hline
\end{tabular}

a The SqET33 line was used as a donor and mRNA containing only an ORF for the transposase was injected into embryos. b The SqET33 line was used as a donor and transposase mRNA containing the $5^{\prime}$ and $3^{\prime}-U T R s$ of the Xenopus $\beta$-globin gene was injected into embryos. ${ }^{c}$ The SqET33-E20 line, a derivative of SqET33, was used as a donor and transposase mRNA containing the 5'- and 3'-UTRs of the Xenopus $\beta$ globin gene was injected into embryos. ${ }^{d}$ Fish with transposon reintegration into germline. The progeny $\left(F_{1}\right)$ of these fish showed new GFP expression patterns different from the original donor one. $e$ Rates are calculated as the percentage of $F_{0}$ founder fish. Since we counted only $F_{0}$ fish producing progeny with new GFP expression patterns, these rates are likely to be underestimates. ${ }^{f}$ Some $F_{0}$ founder fish have more than one insertion in the germline identified by TAIL-PCR.

About $20 \%$ of $\mathrm{F}_{0}$ founder fish produced embryos with the donor-type GFP expression pattern as well as the new GFP expression pattern in the same embryo. In such embryos, donor insertion was always detected by TAIL-PCR, indicating that the donor copy was retained after retransposition. This result suggests that in some cases remobilization probably occurs during DNA replication. Transposition during DNA replication has been well described for other "cut-and-paste" transposons, particularly for $A c / D s[26]$.

\section{Tol2 preferentially reintegrates into linked loci}

By analyzing the chromosomal distribution pattern of the new insertions (see Materials and methods), we found that all chromosomes were hit by Tol2 during re-transposition (Figure 2A and Table 3). About $15 \%$ of reintegration events occurred in the donor chromosomes. Such a linked pattern of re-transposition was found for both donor sites: 23 out of 153 insertions were mapped on chromosome 14 (re-transposition from the SqET33 donor site), while 17 out of 111 were mapped on chromosome 24 (re-transposition from the SqET33-E20 donor site). We also found that about $43 \%(10 / 23)$ and $24 \%(4 / 17)$ of reintegrations occurred less than $1 \mathrm{Mb}$ from the SqET33 and SqET33-E20 donor sites, respectively (Figure 2B). However, these numbers are probably underestimates, since only transpositions that cause changes in GFP expression patterns were considered.

We noticed that some chromosomes were possibly favored targets for Tol2 integration, while others appeared to be disfavored. However, with the exception of the donor chromosomes, only chromosome 11 (when the SqET33 donor site was used for the re-transposition) and chromosome 2 (when the SqET33-E20 donor site was used) were somewhat preferred targets. Chromosome 20 was found to be a potential cold spot for Tol2 integration (when the SqET33-E20 donor site was used for the retransposition). However, hotspots cannot be adequately evaluated at the chromosomal level at a resolution of 338 events. In addition, when all Tol2 integrations were placed on the zebrafish genome map, there was no indication of significant clustering (except on the donor chromosomes). In some cases, two independent transposon insertions were mapped within 3 to $77 \mathrm{~kb}$ of one another (Figure 3).

\section{Tol2 integration into transcription units}

Sequencing of the PCR-amplified regions that flank the target sites from all founder fish confirmed the generation of novel integration events that were not present in the original donor lines (see Additional files 3 and 4). During the three rounds of screening, we isolated 338 transposon integration sites. The genomic positions of 287 of these sites were identified using BLASTN in the Ensembl genome browser (Figure 4A; see Materials and methods for details). However, we could not unambiguously map the remaining 51 integration sites because (i) multiple hits were found that were either very similar or identical to the genomic sequence (26 integration sites), (ii) inte-

Table 2: Number of Tol2 insertions per $F_{0}$ germline

\begin{tabular}{cc}
\hline Number of insertions/ $\mathbf{F}_{\mathbf{0}}$ germline & Number of $\mathbf{F}_{\mathbf{0}}$ founder fish $(\mathbf{n}=\mathbf{2 0 8})$ \\
\hline 1 & $122(59 \%)$ \\
2 & $48(23 \%)$ \\
3 & $27(13 \%)$ \\
4 & $8(4 \%)$ \\
5 & $2(1 \%)$ \\
6 & 0 \\
7 & $1(0.5 \%)$ \\
\hline Donor insertion & $36(17 \%)$
\end{tabular}

\footnotetext{
a $F_{0}$ founder fish were outcrossed to wild type fish and the resulting embryos showing new GFP expression patterns were analyzed by TAIL-PCR. ${ }^{b}$ The donor insertion retained after reintegration was confirmed by TAIL-PCR. These are cumulative data from three rounds of screening.
} 
Table 3: Distribution of Tol2 insertions on chromosomes

\begin{tabular}{|c|c|c|c|c|c|c|}
\hline \multirow[b]{2}{*}{ Chromosome } & \multicolumn{3}{|c|}{ SqET33 donor line $(n=153)$} & \multicolumn{3}{|c|}{ SqET33-E20 donor line $(n=I I I)$} \\
\hline & Number of insertions & $P$-value & $\begin{array}{l}\text { Expected number of } \\
\text { insertions }\end{array}$ & Number of insertions & $P$-value & $\begin{array}{l}\text { Expected number of } \\
\text { insertions }\end{array}$ \\
\hline 1 & 3 & 0.122 & 7 & 2 & 0.17 & 5 \\
\hline 2 & 6 & 1.0 & 6 & 8 & 0.042 & 4 \\
\hline 3 & 9 & 0.44 & 7 & 4 & 0.648 & 5 \\
\hline 4 & 4 & 0.65 & 5 & 3 & 0.611 & 4 \\
\hline 5 & 4 & 0.147 & 8 & 5 & 0.675 & 6 \\
\hline 6 & 7 & 1.0 & 7 & 7 & $0.36 \mathrm{I}$ & 5 \\
\hline 7 & 7 & 0.717 & 8 & 9 & 0.209 & 6 \\
\hline 8 & 5 & 0.44 & 7 & 4 & 0.648 & 5 \\
\hline 9 & 3 & 0.212 & 6 & 2 & 0.309 & 4 \\
\hline 10 & 5 & 1.0 & 5 & 3 & 0.611 & 4 \\
\hline 11 & 10 & 0.023 & 5 & I & 0.127 & 4 \\
\hline 12 & 5 & 0.678 & 6 & 1 & 0.127 & 4 \\
\hline 13 & 5 & 0.678 & 6 & 6 & 0.309 & 4 \\
\hline 14 & 23 & $<0.0001$ & 5 & 3 & 0.361 & 5 \\
\hline 15 & 5 & 1.0 & 5 & 4 & 1.0 & 4 \\
\hline 16 & 9 & 0.212 & 6 & 3 & 0.611 & 4 \\
\hline 17 & 2 & 0.096 & 6 & 5 & 0.611 & 4 \\
\hline 18 & 4 & 0.406 & 6 & 4 & 1.0 & 4 \\
\hline 19 & 7 & 0.364 & 5 & 5 & 0.611 & 4 \\
\hline 20 & 10 & 0.247 & 7 & 0 & 0.022 & 5 \\
\hline 21 & 3 & 0.364 & 5 & 4 & 1.0 & 4 \\
\hline 22 & 5 & 1.0 & 5 & I & 0.242 & 3 \\
\hline 23 & 5 & 1.0 & 5 & 5 & 0.611 & 4 \\
\hline 24 & 5 & 1.0 & 5 & 17 & $<0.0001$ & 3 \\
\hline 25 & 2 & 0.312 & 4 & 5 & 0.242 & 3 \\
\hline Un & 15 & ND & ND & 8 & ND & ND \\
\hline
\end{tabular}

ND, not determined; Un, contig not assigned to any chromosome; $P$-value was calculated by using a $\chi^{2}$ test; $n$, number of mapped insertions.

grations occurred in repetitive dinucleotide sequences (eight integration sites) or (iii) there were sequence gaps in the database (17 sites). Approximately $39 \%$ of the mapped integration sites were found within known or predicted genes annotated in the zebrafish genome. About $82 \%$ of these sites were mapped within introns and about $18 \%$ within exons. There was a bias towards introns because their cumulative size is much larger than that of exons, so they present a much larger target for transposon integration. Assuming a 5-kb interval as an arbitrary threshold for the regulatory regions at the $5^{\prime}$ and 3 ' ends of genes, the frequency of integration was about $62 \%$ for known or predicted transcription units. Furthermore, about 59\% (64/109) of the "intergenic" insertions were mapped within $50-\mathrm{kb}$ regions upstream or downstream of known or predicted genes annotated in the zebrafish genome. We also compared the distribution pattern of insertions depending on donor site. No significant difference was found (Table 4).

We found five genes (ENSDARG00000033473, ENSDARG00000034820, zgc:110750, foxa and fgf13) that were recurrently hit by Tol2. Two insertions in
ENSDARG00000033473 (334 kb) were mapped in one intron with a distance of $41 \mathrm{~kb}$ between the integration sites. Similarly, two insertions in zgc:110750 (196 kb) were mapped in one intron with a distance of $6 \mathrm{~kb}$ between the integration sites. Two insertions in ENSDARG00000034820 (67 kb) were mapped in an intron and $4 \mathrm{~kb}$ downstream of the gene, while insertions in fgf13 (228 kb) were mapped in two different introns. Finally, the two insertions in foxa $(5 \mathrm{~kb})$ were mapped 3 $\mathrm{kb}$ upstream and $1 \mathrm{~kb}$ downstream from the gene. Four of these five genes (fgf13 was the exception) were hit from both independent donor sites. Since the sizes of the targeted genes differ substantially (from $5 \mathrm{~kb}$ to $334 \mathrm{~kb}$ ), the distance between two independently integrated Tol2 transposons is more important (in these cases, the distance ranges from $6 \mathrm{~kb}$ to $47 \mathrm{~kb}$ ). We calculated the probability of hitting a similarly-sized locus in the zebrafish genome and then used a binomial distribution test to determine the statistical significance of each targeted locus being hit twice. The P values ranged from $3.3 \times 10-6$ to 2.8 $\times 10-3$, probably indicating potential hotspots within these genes (with an exception of fgf13, since it is linked to the donor site). 
A
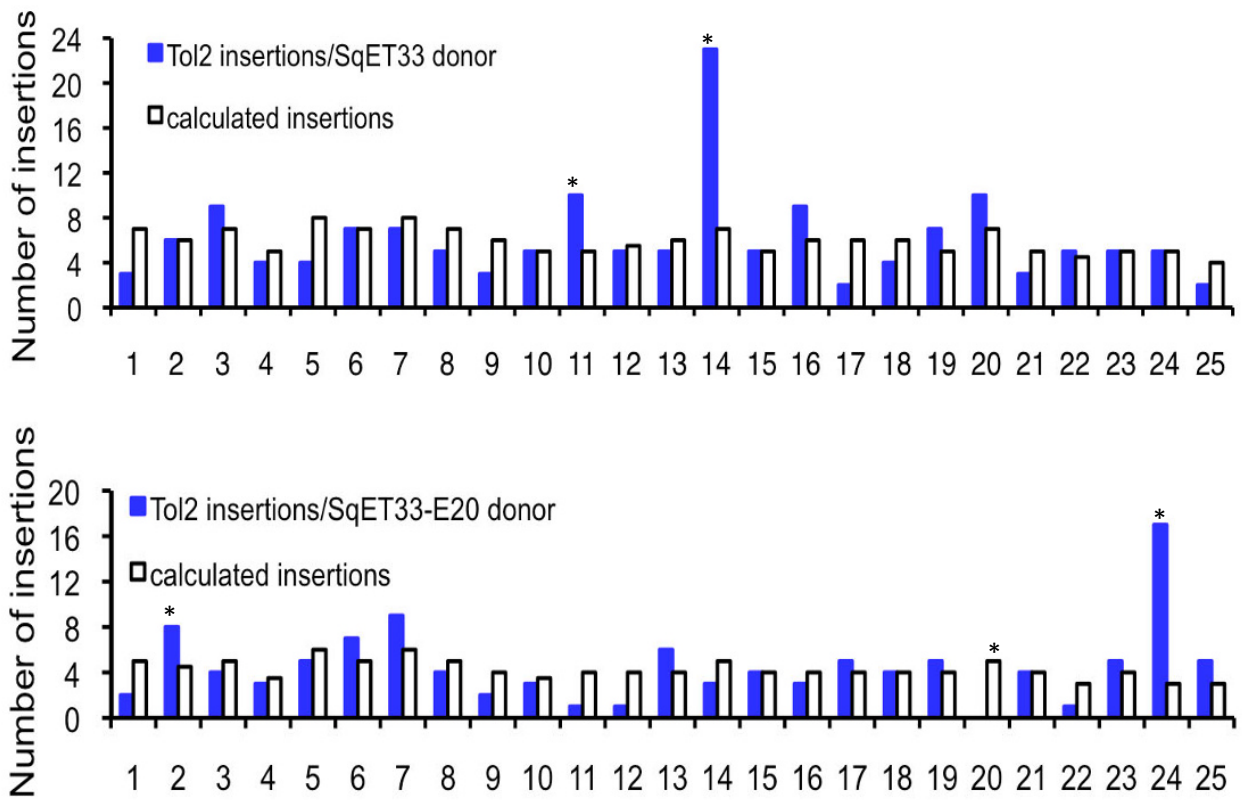

Chromosomes

B
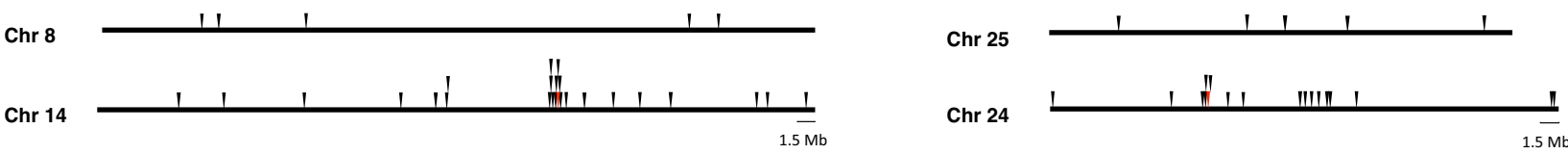

Figure 2

Genome-wide distribution pattern of Tol2 integrations. A: Chromosomal distribution pattern of Tol2 insertions depending on the donor site. Asterisks show statistically significant differences $(p<0.000$ I for the donor chromosomes and $p$ $<0.05$ for the others). B: Pattern of Tol2 reintegrations linked to the different donor sites (chromosomes 14 and 24). Nondonor chromosomes 8 and 25 , comparable in size to the donor chromosomes, are shown for reference. Red triangles represent the donor sites.

\section{Tol 2 integration into endogenous repeat elements}

We analyzed the distribution pattern of Tol2 insertions with respect to various genomic repeat elements (Table 5); 128 out of the 338 integration sites were found in endogenous repeat elements that are currently annotated in the zebrafish genome. Most of these targeted repeat elements belong to DNA transposons and to unclassified Dr repeats. In addition, our results showed that Tol2 was less prone to integrate into retrotransposons (17 integration events) and tandem repeats (16 integration events). Interestingly, LTR-containing retrotransposons were very seldom targeted by Tol2 (only 3 integration events). According to Repbase Update [27], 357 different families of DNA transposons and 279 of retrotransposons are currently annotated in the zebrafish genome. Retrotrans- posons that contain LTRs are more diversely represented in the zebrafish genome than those that do not (222 vs. 57 families). The differences in targeting of repetitive elements observed in our experiment could be explained by a difference in either the copy number or the global distribution pattern of each class of repetitive elements in the zebrafish genome.

\section{Specificity of Tol 2 integration site}

Some transposable elements exhibit a high degree of integration specificity, while others display relatively little preference for a target DNA sequence [28,29]. We analyzed the nucleotide composition over a 48-bp sequence region comprising an 8 -bp target site and 20-bp flanking sequences on each side according to [30] (Figure 4B). In 


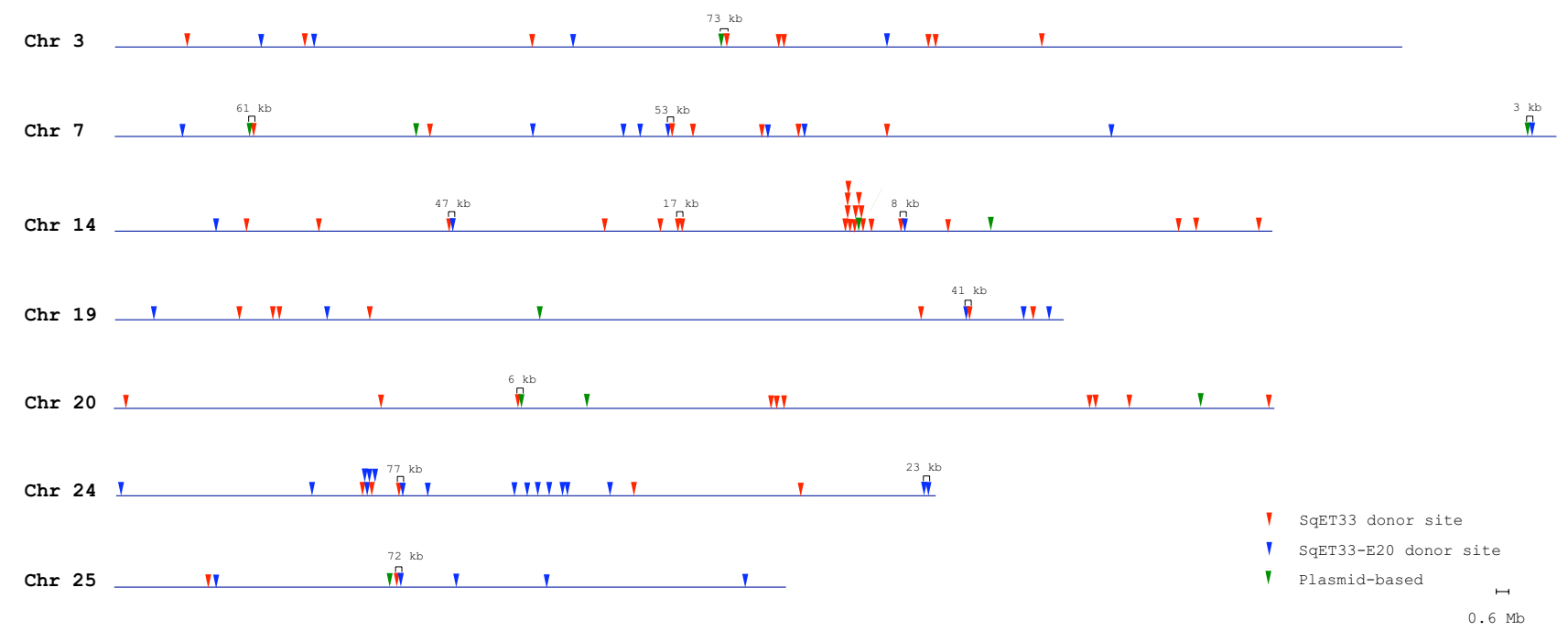

\section{Figure 3}

Clustering of Tol2 integrations in the zebrafish genome. Only chromosomes in which two independent Tol2 insertions are mapped within less than $100-k b$ of one another are shown. Data for the plasmid-based derived integrations were taken from [13] and remapped.

addition to the 329 integration sites isolated in this study, we also included 39 integration sites isolated from our previous ET screen [13]. Comparisons among all the Tol2 integration sites and flanking DNA revealed no conserved pattern in the sequences flanking the eight base pair duplication at the integration site. The only exceptions were the nucleotides at \pm 3 and \pm 1 bp relative to the integration site, which were $46 \%$ and $40 \%$ conserved, respectively (see Additional file 5). In addition, we detected a weak AT-rich consensus that contained a palindrome-like core sequence TNA(C/G)TTATAA(G/C)TNA centered at the insertion site (shown in bold). However, only four integration sites were actual palindromes.

\section{Discussion}

In this study, we analyzed a genome-wide reintegration of the non-autonomous transposable element Tol2 in zebrafish when it was remobilized from two different donor sites. We showed that the genomic Tol2 copy can be remobilized upon injection of transposase mRNA into the germlines of up to $48 \%$ of founders. Since we selected only those Tol2 reintegrations in germlines that caused changes in GFP expression, we in fact measured the "apparent transposition rate"; the actual germline transposition frequency during in vivo remobilization would be higher.

We analyzed Tol2 integration sites with respect of their chromosomal distribution, integration into intragenic regions and insertion site sequence specificity. Although novel integration sites were found on different chromosomes, Tol2 reintegration was not random. Almost $39 \%$ of transposon integrations were found within known or predicted genes. Most of them were found within introns, as expected in view of the high intron/exon ratio in the zebrafish genome. If we consider insertions into the regulatory regions adjacent to transcriptional initiation and termination sites, the rate of Tol2 transposition into genes was even higher. Since we used the TAIL-PCR method to isolate transposon inserts, we could not recover all possible Tol2 insertions in the genome. However, despite of using enhancer trap approach, the frequency with which Tol2 was integrated within intragenic regions was similar to that found for the $S B$ transposon in human $(39 \%)$ and mouse (31\%) cells [31] and for $A c / D s$ in rice (30\%) [3234] and Arabidopsis (38\%) [35]. Therefore, Tol2 is as prone to integrate into transcriptional units as other DNA transposons.

Our results further demonstrated that about $15 \%$ of Tol2 reintegrations from a specific donor site were linked to the same chromosome. Such behavior was also noticed in [11], where about $18 \%$ of the mapped integration sites (6/ $34)$ were located on the donor chromosome. We found that about one third of intrachromosomal reintegrations were located within $1 \mathrm{Mb}$ of the donor site. However, since we selected reintegrations on the basis of new GFP expression patterns, this number is likely to be lower than the actual number of such transpositions (for example, closely-linked transpositions may retain the GFP expres- 
A

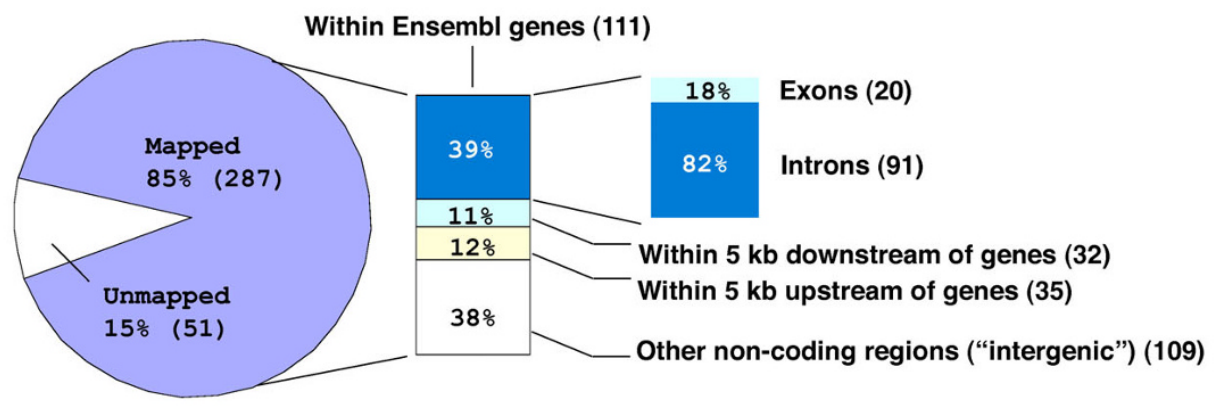

B

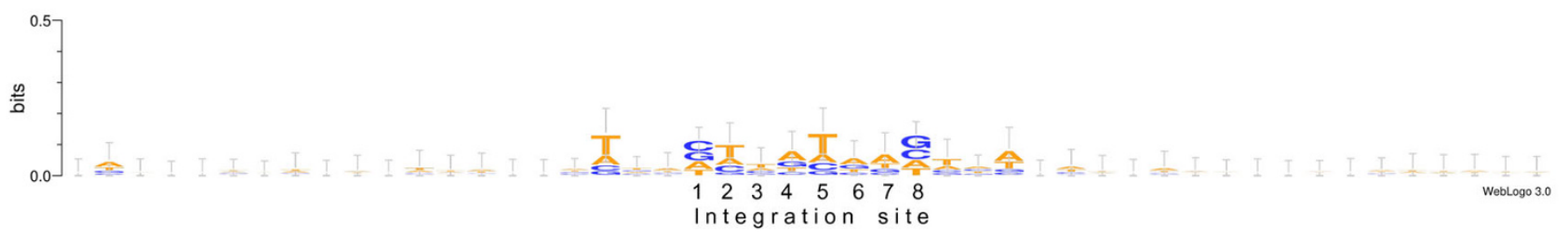

\section{Figure 4}

Tol2 integration properties. A: Distribution of Tol2 insertions in respect of endogenous genes. B: Tol2 integration site motif analysis. A graphical representation of a nucleic acid multiple sequence alignment $(n=368)$ was generated by WebLog (version 3.0).

sion pattern of the donor). This local hopping phenomenon has been described for other DNA transposons $[19,36-40]$. For example, $S B$ is mostly re-integrated within $3 \mathrm{Mb}$ of the donor site [19] and the local hopping interval of the $P$ element is within $100 \mathrm{~kb}$ [36]. Local hopping was also found for the hAT family. In this case, more than half of $A c$ transposon reintegrations occurred within $1.7 \mathrm{Mb}$ of the donor site [37]. Overall, a linked reintegration prop- erty of the Tol2 system might be beneficial for setting a region-specific saturation ET screen.

Interestingly, our analysis revealed that some chromosomes other than the donor were somewhat preferred targets for Tol2 integration; still others appeared to be disfavored. Such transposon behavior may reflect the spatial chromosomal architecture within the nucleus, if mul-

Table 4: Distribution of Tol2 insertions depending on donor site

\begin{tabular}{|c|c|c|c|c|}
\hline \multirow[t]{2}{*}{ Donor site ${ }^{a}$} & \multicolumn{2}{|c|}{ SqET33 donor } & \multicolumn{2}{|c|}{ SqET33-E20 donor } \\
\hline & Number & Frequency $^{\text {b }}$ & Number & Frequency $^{b}$ \\
\hline Insertions in introns & 50 & $30 \%$ & $4 I$ & $34 \%$ \\
\hline Insertions in exons & 15 & $9 \%$ & 5 & $4 \%$ \\
\hline Insertions in $5^{\prime}$ endc & 20 & $12 \%$ & 15 & $12 \%$ \\
\hline Insertions in $3^{\prime}$ endc & 22 & $13 \%$ & 10 & $8 \%$ \\
\hline Intergenic insertions ${ }^{d}$ & 59 & $36 \%$ & 50 & $41 \%$ \\
\hline Mapped insertions & 166 & & $|2|$ & \\
\hline
\end{tabular}

a For SqET33, donor site data from the Ist and 2nd screens were combined. b Frequencies are calculated as the percentages of particular insertions. c We set a 5-kb interval as an arbitrary threshold for a regulatory region at the 5' and 3' ends of a transcription unit. d More than $5 \mathrm{~kb}$ upstream or downstream of a transcription unit. Insertions with no annotated tagged gene were also considered "intergenic". 
Table 5: Tol2 integrations into genomic repetitive elements

\begin{tabular}{cc}
\hline Repetitive elements & Number of insertions \\
\hline DNA transposon & 48 \\
Dr element & 47 \\
non-LTR retrotransposon & 14 \\
LTR retrotransposon & 3 \\
Tandem repeat & 16 \\
All genomic repeats & $128(38 \%)$ \\
Sequenced insertions & 338
\end{tabular}

a Include LINE, long interspersed nuclear element; SINE, short interspersed nuclear element. $b$ Include microsatellites and minisatellites. These are cumulative data from three rounds of screening.

tiple non-adjacent chromosome segments are closely juxtaposed at the nuclear interior or periphery [41-43]. There are many examples of correlations among the intranuclear positions of genes, their clusters and genetic activities, whereas the relative positioning of chromosomes seems to be maintained (reviewed in [44]). Therefore, such a property of transposons may potentially be used for analyzing the spatial organization of the genome.

We found that Tol2 differentially targeted the different classes of endogenous repetitive elements. For example, it more frequently targeted DNA transposons than retrotransposons and tandem repeats. The latter tendency contrasts with the profound preference of Tc1/mariner transposons for TA-containing microsatellite DNA $[31,45]$. Expansion of such repeats during replication slippage can cause repeat instability and increased recombination rates (reviewed in [46]), suggesting that these transposons may use the recombination machinery during integration. Like the $S B$ transposon, Tol2 also avoided retrotransposons containing LTRs. The differences in targeting of endogenous repetitive elements may reflect differences in the copy numbers of each class of repeats, as well as differences among the mechanisms of integration utilized by each transposon family.

We also found that Tol2 was prone to integrate into ATrich DNA regions and that a target site contained a weak palindrome-like consensus sequence. An AT-rich palindromic consensus has previously been found in the target sequence of Tc1/mariner transposons such as $S B$ in human and mouse cells [31] and the Tc1 element in worms [47]. However, in contrast to the strict preference of $S B$ and Tc1 for TA dinucleotide targets, Tol2 has no such preference at the nucleotide level. There is some evidence that distinct preferred transposon integration sites may not necessary match consensus sequences, but rather share similar structural patterns [48]. DNA structural characteristics such as bending and protein-induced deformability play an important role in directing DNA integration $[28,48,49]$. DNA bending can lead to changes in the width and depth of the major and minor grooves, affecting a protein's access [49]. AT-rich palindromes are particularly susceptible to local melting and have been experimentally shown to adopt a bendable DNA structure [50]. In addition, palindromic sequences have the potential to form cruciform configurations, which are an efficient target for RAGmediated transposition [51]. Also, AT-rich palindromic repeats are known to be double-strand break hotspots. It has been proposed that DNA bending plays a role in the integration specificity of the hobo transposable element from the hAT family, but hobo has no strict preference for targeting at nucleotide level [39]. This suggests the likelihood that the target site selection of Tol2 is primarily determined at the level of DNA structure, not sequence.

Tol2 element transposes by "cut-and-paste" mechanism, which involves the excision and re-integration of the transposon from one site to another, creating an 8-bp duplication of the integration site $[3,13]$. Previously, we found that in one third of Tol2 excision events, reparation of donor site results in different footprints [13]. In our experiments we used extremely high amounts of transposase mRNA (around $9 \times 10^{7}$ molecules per a single copy of transposon), therefore it may be reasonable to expect multiple "cut-and-paste" events before transposon will finally settle. Such multiple hops could generate doublestrand breaks and, as a consequence, the footprints. Our analysis of DNA sequences flanking the integration/target site revealed no signs of the footprints, at least, at the vicinity (up to $900 \mathrm{bp}$ ) of new integration sites. All DNA sequence modifications found at these regions exhibited DNA sequence polymorphism between zebrafish strains (data not shown). However, we could not rule out the possibility that the footprints left after multiple "cut-andpaste" events may be found far away from integration sites.

\section{Conclusion}

In summary, in a large-scale ET screen we analyzed 338 insertions generated in the zebrafish genome by remobilization of a single Tol2 transposon copy from two independent sites on two different chromosomes. About 39\% of Tol2 insertions occurred within genes, mostly in introns. Upon remobilization, Tol2 showed a preference to reintegrate within the chromosome containing the donor site. Sequence analysis of integration sites revealed no strict specificity at the nucleotide level, but Tol2 was prone to integrate into AT-rich regions with weak palindrome-like consensus sequences. This information should be carefully evaluated during the design of various follow-up applications that involve Tol2. Numerous ET lines with diverse GFP expression patterns have been generated in this work. They represent a large set of research tools for in vivo studies of vertebrate development, and some have already been successfully used for that purpose $[22,52,53]$. 


\section{Methods \\ Fish lines}

The ET(krt4:EGFP) ${ }^{\mathrm{SqET33}}$ line (referred to as SqET33) was established by coinjection of in vitro synthesized transposase mRNA and Tol2 transposon-based ET construct DNA into wild type zebrafish embryos at the one- or two-

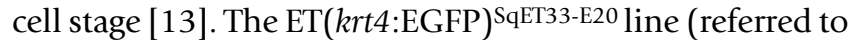
as SqET33-E20) was created after in vivo remobilization of a single-copy of Tol2 transposon-based ET construct from the donor line SqET33 [22]. Fish were maintained according to established protocols [54] and in agreement with the IACUC regulations and rules of the IMCB zebrafish facility. Transgenic and wild type fish were $A B$ strain.

\section{Plasmids}

Plasmid pTem03 containing the coding region for medaka Tol2 transposase was purchased from Dr. Koga (Nagoya University, Japan) as a part of the "Gene transfer system" kit. Plasmid pDB600 containing the coding region of Tol 2 transposase flanked with the $5^{\prime}$ and 3' UTRs from the Xenopus $\beta$-globin gene was kindly provided by Dr. Ekker (University of Minnesota, USA).

\section{In vitro $m R N A$ synthesis and in vivo transposon remobilization}

Plasmids pTem03 and pDB600 were linearized with XbaI and SpeI, respectively, and used as templates for in vitro mRNA synthesis. Transposase mRNA was synthesized using mMESSAGE mMACHINE SP6 and T3 kits (Ambion, USA) and purified using an RNeasy Mini Kit (QIAGEN, Germany). For in vivo remobilization of a single-copy of Tol2 transposon-based ET construct, 50-100 pg of transposase mRNA was injected into zebrafish embryos from the donor lines at the one- or two-cell stage.

\section{Southern blot hybridization}

Genomic DNA from adult fish or embryos was phenol extracted and digested using HindIII (New England Biolabs), which cut the Tol2 transposon-based ET cassette at a unique site. The digested genomic DNA was fractionated by agarose gel electrophoresis, transferred to a positively charged nylon membrane (Hybond- $\mathrm{N}^{+}$, Amersham Biosciences) by capillary blotting [55], and crosslinked by UV irradiation. The DNA probe for EGFP was labeled with digoxigenin (DIG) using a PCR DIG synthesis kit (Roche Applied Science). We used DIG EasyHyb buffer, an antiDIG alkaline phosphatase conjugate antibody and CDPStar chemiluminescent substrate (all Roche Applied Science) to detect the hybridized probe. Hybridization and detection were carried out according to the manufacturer's instructions.

\section{Identification and mapping of integration sites}

To recover genomic sequences flanking the integrated Tol2 transposon we used thermal asymmetric interlaced PCR
(TAIL-PCR). The DNA was isolated from one GFP-positive embryo after outcrossing of $\mathrm{F}_{0}$ fish with wild type fish. TAIL-PCR was performed according to Liu and Whittier [56] using the primers and cycling conditions described elsewhere [13]. The resulting PCR products were purified and directly sequenced using primers 5'CCCCAAAATAATACTTAAGTACAG-3' and 5'-GTACTTGTACTTTCACTTGAG-3', which anneal to the 5' and 3' transposon ends, respectively. The length of the sequence reads never exceeded $900 \mathrm{bp}$. A sequence was considered to be from an authentic integration site only if it contained the Tol2 transposon sequence from the nested primer to the ends of the inverted repeats. In total, we amplified and sequenced 519 genomic regions flanking the 338 integration sites (see Additional file 4 for details). Of these, 159 sequences were amplified from either the $5^{\prime}$ or the $3^{\prime}$ end of the integrated transposon only. The sequence reads were then mapped to the zebrafish genome using BLASTN (the latest zebrafish whole genome assembly version 7 (Zv7, April 2007 freeze in the Ensembl genome browser) http://www.ensembl.org. In some cases, the flanking sequences were blasted against the unfinished high-throughput genomic sequences (htgs) database or trace archive at NCBI http:// www.ncbi.nlm.nih.gov. We considered the sequence to be from a unique integration site if it matched to no more than one genomic locus with $95 \%$ or greater identity to the genomic sequence over the high-quality sequence region (a whole length of sequence read). On the basis of this criterion, 423 sequences reads from 275 integration sites could be unambiguously mapped to unique genomic loci. Sixty sequence reads from 38 integration sites were matched to more than one genomic locus with $95 \%$ or greater identity to the genomic sequence. In those cases, only the hits with highest identity (>99\%) and score over the whole length of sequence read were considered. Thus, 20 sequence reads from 12 integration sites were unmistakably mapped to distinct genomic loci. The remaining 40 sequence reads from 26 integration sites could not be mapped unambiguously. We also had 26 sequence reads from 17 integration sites that were either matched with less than $95 \%$ of identity to the genomic sequence, or had no significant similarity to the genomic database. Half the sequence reads that had no hits in the latest version of the database ( $\mathrm{Zv} 7)$ were matched to a single genomic locus with $98 \%$ or greater identity to the genomic sequence over the sequence region in the previous zebrafish whole genome assembly, version Zv6. Nevertheless, such sequence reads were not considered to be matched. In addition, 10 sequences from 8 integration sites represented short $(<100 \mathrm{bp})$ repetitive dinucleotide sequence reads that could not be mapped to any location. Ultimately, we were able to map 287 integration sites out of 338 integration events to unique genomic loci. 


\section{Bioinformatics}

We defined integration as having landed in a gene only if it was within the genomic coordinates of the 21,322 protein-coding genes or transient EST gene models annotated in the zebrafish genome. The 5' and 3' ends of the genes were considered the first and last nucleotide positions according to gene coordinates in the latest zebrafish whole genome assembly, Zv7. We analyzed the base composition over a 48-bp region encompassing the Tol2 target site using the computer program WebLogo (version 3.0) http://weblogo.threeplusone.com. We also analyzed integrations in relation to various genomic repeat elements annotated in the zebrafish genome. The expected number of insertions in each chromosome (if integration events were random) was calculated as follows: (1) to calculate the expected distribution of insertions, the total number of bases in the zebrafish genome was divided by the number of mapped insertions; (2) to calculate the expected number of insertions on each chromosome, the length of each individual chromosome was divided by the expected distribution of insertions. The total number of base pairs in the zebrafish genome (reference assembly length) according to the Zv7 assembly is 1,440,582,308 base pairs. Integration into chromosomes was tested for statistical bias using a $\chi^{2}$ test to compare the observed number of integrations into a particular chromosome to the value expected if integration events were random.

\section{List of abbreviations}

ET: enhancer trap; GFP: green fluorescent protein; EGFP: enhanced GFP; LTR: long terminal repeats; ORF: open reading frame; SB: Sleeping Beauty transposon; TAIL-PCR: thermal asymmetric interlaced PCR; UTR: untranslated region; WT: wild type; bp: base pairs.

\section{Authors' contributions}

Conceived and designed the experiments: IK, AE, SP and VK. Performed the experiments: IK and MGL. Analyzed the data: IK, MGL, AE, SP and VK. Wrote the manuscript: IK, SP and VK. All authors read and approved the final manuscript.

\section{Additional material}

\section{Additional file 1}

GFP segregation in $F_{1}$ generation. Table $S 1$ shows the number of novel expression patterns and GFP segregation ratios in the $F_{1}$ generation.

Transgenic embryos $\left(F_{0}\right)$ carrying a single heterozygous Tol 2 insert in their genome $\left(\mathrm{gf} \mathrm{p}^{+-}\right)$were injected with transposase mRNA, raised to maturity, and outcrossed to wild-type fish.

Click here for file

[http://www.biomedcentral.com/content/supplementary/14712164-10-418-S1.pdf]

\section{Additional file 2}

Southern blot hybridization of $F_{1}$ generation. Figure $S 1$ shows the Tol2 copy number in $F_{1}$ fish. DNA was isolated from individual $F_{1}$ fish that originated from the same $F_{0}$ founder. The DNA samples were digested with HindIII and hybridized with DIG-labeled EGFP probe.

Click here for file

[http://www.biomedcentral.com/content/supplementary/14712164-10-418-S2.pdf]

\section{Additional file 3}

Integration sites and genes located near the insertions. Table S2 shows the integration loci and nearest genes targeted by Tol2 remobilized from two different donor sites. Targeted repetitive elements are also shown. Click here for file

[http://www.biomedcentral.com/content/supplementary/14712164-10-418-S3.pdf]

\section{Additional file 4}

Flanking genomic sequences of Tol 2 transposon insertions. Table S3 shows the DNA sequence reads of the regions that flank Tol2 transposon insertions. To obtain the genomic locations of Tol2 insertions, the flanking sequence reads were blasted against the latest zebrafish genome sequence database (zebrafish whole genome assembly, version 7) from the Ensembl genome browser http://www.ensembl.org. In some cases, the flanking sequence reads were blasted against the unfinished high-throughput genomic sequence (htgs) database or trace archive at NCBI http:// www.ncbi.nlm.nih.gov.

Click here for file

[http://www.biomedcentral.com/content/supplementary/14712164-10-418-S4.pdf]

\section{Additional file 5}

Target site analysis for 368 genomic Tol2 insertions. Table S4 shows the base composition of an 8-bp integration site in 368 genomic Tol2 insertions. For the region flanking the integration site only the first three nucleotides are shown.

Click here for file

[http://www.biomedcentral.com/content/supplementary/14712164-10-418-S5.pdf]

\section{Acknowledgements}

We thank the personnel of the IMCB fish facility for the maintenance of the fish lines, and the personnel of the IMCB DNA sequence facility for the sequencing. This work was financially supported by the Agency for Science, Technology and Research (A-STAR) of Singapore. The authors declare absence of conflicting financial interests.

\section{References}

I. Koga A, Suzuki M, Inagaki H, Bessho Y, Hori H: Transposable element in fish. Nature 1996, 383:30.

2. Koga A: Transposition mechanisms and biotechnology applications of the medaka fish Tol2 transposable element. Adv Biophys 2004, 38:161-180.

3. Kawakami K, Shima A, Kawakami N: Identification of a functional transposase of the Tol2 element, an Ac-like element from the Japanese medaka fish, and its transposition in the zebrafish germ lineage. Proc Natl Acad Sci USA 2000, 97: I | 403-I I 408. 
4. Kawakami K, Noda T: Transposition of the Tol2 element, an Aclike element from the Japanese medaka fish Oryzias latipes, in mouse embryonic stem cells. Genetics 2004, 166:895-899.

5. Kawakami K: Transposon tools and methods in zebrafish. Dev Dyn 2005, 234:244-254.

6. Balciunas D, Wangensteen KJ, Wilber A, Bell J, Geurts A, Sivasubbu S, Wang X, Hackett PB, Largaespada DA, Mclvor RS, Ekker SC: Harnessing a high cargo-capacity transposon for genetic applications in vertebrates. PLoS Genet 2006, 2: el69.

7. Fisher S, Grice EA, Vinton RM, Bessling SL, Urasaki A, Kawakami K, McCallion AS: Evaluating the biological relevance of putative enhancers using Tol2 transposon-mediated transgenesis in zebrafish. Nat Protoc 2006, I: |297-|305.

8. Hamlet MR, Yergeau DA, Kuliyev E, Takeda M, Taira M, Kawakami K, Mead PE: Tol2 transposon-mediated transgenesis in Xenopus tropicalis. Genesis 2006, 44:438-445.

9. Davison JM, Akitake CM, Goll MG, Rhee JM, Gosse N, Baier H, Halpern ME, Leach SD, Parsons MI: Transactivation from Gal4-VP I 6 transgenic insertions for tissue-specific cell labeling and ablation in zebrafish. Dev Biol 2007, 304:8 I I-824.

10. Scott EK, Mason L, Arrenberg AB, Ziv L, Gosse NJ, Xiao T, Chi NC Asakawa K, Kawakami K, Baier H: Targeting neural circuitry in zebrafish using GAL4 enhancer trapping. Nat Methods 2007 , 4:323-326

I I. Urasaki A, Azakawa K, Kawakami K: Efficient transposition of the Tol2 transposable element from a single-copy donor in zebrafish. Proc Natl Acad Sci USA 2008, I 05: I9827-19832.

12. Kawakami K, Takeda H, Kawakami N, Kobayashi M, Matsuda N, Mishina $M$ : A transposon-mediated gene trap approach identifies developmentally regulated genes in zebrafish. Dev Cell 2004, 7:133-144

13. Parinov S, Kondrichin I, Korzh V, Emelyanov A: Tol2 transposonmediated enhancer trap to identify developmentally regulated zebrafish genes in vivo. Dev Dyn 2004, 23 I:449-459.

14. Asakawa K, Suster ML, Mizusawa K, Nagayoshi S, Kotani T, Urasaki A, Kishimoto Y, Hibi M, Kawakami K: Genetic dissection of neural circuits by Tol2 transposon-mediated Gal4 gene and enhancer trapping in zebrafish. Proc Natl Acad Sci USA 2008 , 105: | 255-1260.

15. Sivasubbu S, Balciunas D, Davidson A, Pickart M, Hermanson S, Wangensteen K, Wolbrink D, Ekker S: Gene-breaking transposon mutagenesis reveals an essential role for histone $\mathrm{H} 2 \mathrm{afza}$ in zebrafish larval development. Mech Dev 2006, I 23:5 I3-529.

16. Nagayoshi S, Hayashi E, Abe G, Osato N, Asakawa K, Urasaki U, Horikawa K, Ikeo K, Takeda H, Kawakami K: Insertional mutagenesis by the Tol2 transposon-mediated enhancer trap approach generated mutations in two developmental genes: tcf7 and synembryn-like. Development 2008, 135:159-169.

17. Korzh V: Transposons as tools for enhancer trap screens in vertebrates. Genome Biol 2007, 8:S8

18. Halpern ME, Rhee J, Goll MG, Akitake CM, Parsons M, Leach SD Gal4/UAS transgenic tools and their application to zebrafish. Zebrafish 2008, 5:97-110.

19. Horie K, Yusa K, Yae K, Odajima J, Fischer SE, Keng VW, Hayakawa T, Mizuno S, Kondoh G, ljiri T, Matsuda Y, Plasterk RH, Takeda J: Characterization of Sleeping Beauty transposition and its application to genetic screening in mice. Mol Cell Biol 2003, 23:9189-9207.

20. Keng VW, Yae K, Hayakawa T, Mizuno S, Uno Y, Yusa K, Kokubu C, Kinoshita T, Akagi K, Jenkins NA, Copeland NG, Horie K, Takeda J: Region-specific saturation germline mutagenesis in mice using the Sleeping Beauty transposon system. Nat Methods 2005, 2:763-769.

21. Choo BG, Kondrichin I, Parinov S, Emelyanov A, Go W, Toh WC Korzh V: Zebrafish transgenic Enhancer TRAP line database (ZETRAP). BMC Dev Biol 2006, 6:5

22. García-Lecea M, Kondrychyn I, Fong $\mathrm{SH}$, Ye ZR, Korzh V: In vivo analysis of choroid plexus morphogenesis in zebrafish. PLoS ONE 2008, 3:e3090

23. Emelyanov A, Gao Y, Naqvi NI, Parinov S: Trans-kingdom transposition of the maize Dissociation element. Genetics 2006, 174:1095-1104.

24. Grevelding C, Becker D, Kunze R, von Menges A, Fantes V, Schell J Masterson R: High rates of Ac/Ds germinal transposition in Arabidopsis suitable for gene isolation by insertional mutagenesis. Proc Natl Acad Sci USA 1992, 89:6085-6089.
25. Gorbunova V, Levy A: Circularized Ac/Ds transposons: formation, structure and fate. Genetics 1997, I45: I I6I-I I69.

26. Fedoroff NV: About maize transposable elements and development. Cell 1989, 56:|8|-|9|.

27. Jurka J, Kapitonov VV, Pavlicek A, Klonowski P, Kohany O, Walichiewicz J: Repbase Update, a database of eukaryotic repetitive elements. Cytogenet Genome Res 2005, I I 0:462-467.

28. Handler AM: Use of the piggyBac transposon for germ-line transformation of insects. Insect Biochem Mol Biol 2002 32: $1211-1220$.

29. Liu G, Geurts AM, Yae K, Srinivasan AR, Fahrenkrug SC, Largaespada DA, Takeda J, Horie K, Olson WK, Hackett PB]: Target-site preferences of Sleeping Beauty transposons. J Mol Biol 2005, 346: $161-173$

30. Schneider TD, Stephens RM: Sequence logo: a new way to display consensus sequences. Nucleic Acids Res 1990, I 8:6097-6100.

31. Yant SR, Wu X, Huang Y, Garrison B, Burgess SM, Kay MA: Highresolution genome-wide mapping of transposon integration in mammals. Mol Cell Biol 2005, 25:2085-2094.

32. Enoki H, Izawa T, Kawahara M, Komatsu M, Koh S, Kyozuka J, Shimamoto K: Ac as a tool for the functional genomics of rice. Plant J 1999, 19:605-613.

33. Greco R, Ouwerkerk PBF, Taal AJC, Favalli C, Beguiristain T, Puigdomenech P, Colombo L, Hoge JHC, Pereira A: Early and multiple Ac transposition in rice suitable for efficient insertional mutagenesis. Plant Mol Biol 200I, 46:2I 5-227.

34. Greco R, Ouwerkerk PBF, de Kam RJ, Salland C, Favalli C, Colombo L, Guiderdoni E, Meijer AH, Hoge JHC, Pereira A: Transpositional behavior of an $A c / D s$ system for reverse genetics in rice. Theor Appl Genet 2003, I 08: I0-24.

35. Parinov S, Sevugan M, Ye D, Yang WC, Kumaran M, Sundaresan V: Analysis of flanking sequences from Dissociation insertion lines: a database for reverse genetics in Arabidopsis. Plant Cell 1999, I I:2263-2270.

36. Tower J, Karpen GH, Craig N, Spradling AC: Preferential transposition of Drosophila $P$ elements to nearby chromosomal sites. Genetics 1993, I 33:347-359.

37. Machida C, Onouchi H, Koizumi J, Hamada S, Semiarti E, Torikai S, Machida $Y$ : Characterization of the transposition pattern of the Ac element in Arabidopsis thaliana using endonuclease IScel. Proc Natl Acad Sci USA 1997, 94:8675-8680.

38. Luo G, Ivics Z, Izsvak Z, Bradley A: Chromosomal transposition of a Tc I/mariner-like element in mouse embryonic stem cells. Proc Natl Acad Sci USA 1998, 95: I0769-10773.

39. Saville KJ, Warren WD, Atkinson PW, O'Brochta DA: Integration specificity of the hobo element of Drosophila melanogaster is depend on sequences flanking the integration site. Genetica 1999, 105:133-147.

40. Drabek D, Zagoraiou L, de Wit T, Langeveld A, Roumpaki C, Mamalaki C, Savakis C, Grosveld F: Transposition of the Drosophila hydei Minos transposon in the mouse germ line. Genomics 2003, 81:108-I II.

4I. Parreira L, Telhada M, Ramos C, Hernandez R, Neves H, Carmo-Fonseca $M$ : The spatial distribution of human immunoglobulin genes within the nucleus: evidence for gene topography independent of cell type and transcriptional activity. Hum Genet 1997, 100:588-594

42. Skalníková M, Kozubek $S$, Lukásová E, Bártová E, Jirsová $P$, Cafourková A, Koutná I, Kozubek M: Spatial arrangement of genes, centromeres and chromosomes in human blood cell nuclei and its changes during the cell cycle, differentiation and after irradiation. Chromosome Res 2000, 8:487-499.

43. Lukásová E, Kozubek S, Kozubek M, Falk M, Amrichová J: The 3D structure of human chromosomes in cell nuclei. Chromosome Res 2002, 1 0:535-548.

44. Baxter J, Merkenschlager M, Fisher AG: Nuclear organisation and gene expression. Curr Opin Cell Biol 2002, I 4:372-376.

45. Rizzon C, Martin E, Marais G, Duret L, Segalat L, Biemont C: Patterns of selection against transposons inferred from the distribution of Tcl, Tc3 and Tc5 insertions in the mut-7 line of the nematode Ceanorhabditis elegans. Genetics 2003, 1 65: I 127-1/35

46. Ellegren $\mathrm{H}$ : Microsatellites: simple sequences with complex evolution. Nat Rev Genet 2004, 5:435-445

47. Korswagen HC, Durbin RM, Smits MT, Plasterk RH: Transposon Tcl-derived, sequence-tagged sites in Caenorhabditis elegans 
as markers for gene mapping. Proc Natl Acad Sci USA 1996, 93:14680-14685.

48. Geurts AM, Hackett CS, Bell JB, Bergemann TL, Collier LS, Carlson CM, Largaespada DA, Hackett PB: Structure-based prediction of insertion-site preferences of transposons into chromosomes. Nucleic Acids Res 2006, 34:2803-28II.

49. Brukner I, Sanchez R, Suck D, Pongor S: Sequence-dependent bending propensity of DNA as revealed by DNase I: parameters for trinucleotides. $E M B O \mathrm{~J}|995,14:| 8|2-18| 8$.

50. Vigdal TJ, Kaufman CD, Izsvak Z, Voytas DF, Ivics Z: Common physical properties of DNA affecting target site selection of Sleeping Beauty and other Tcl/mariner transposable elements. J Mol Biol 2002, 323:44I -452.

5I. Lee GS, Neiditch MB, Sinden RR, Roth DB: Targeted transposition by the V(D)J recombinase. Mol Cell Biol 2002, 22:2068-2077.

52. Ke Z, Kondrichin I, Gong Z, Korzh V: Combined activity of the two Gli2 genes of zebrafish play a major role in Hedgehog signaling during zebrafish neurodevelopment. Mol Cell Neurosci 2008, 37:388-40I.

53. Vasilyev A, Liu Y, Mudumana S, Mangos S, Lam P, Majumdar A, Zhao J, Poon KL, Kondrychyn I, Korzh V, Drummond I: Collective cell migration drives morphogenesis of the kidney nephron. PLOS Biol 2009, 7:e9.

54. Westerfield M: The Zebrafish Book Eugene, University of Oregon Press; 1993.

55. Sambrook J, Fritsch EF, Maniatis T: Molecular Cloning: A Laboratory Manual Cold Spring Harbor, Cold Spring Harbor Press; 1989.

56. Liu YG, Whittier RF: Thermal asymmetric interlaced PCR: automatable amplification and sequencing of insert end fragments from PI and YAC clones for chromosome walking. Genomics 1995, 25:674-68I.

Publish with Bio Med Central and every scientist can read your work free of charge

"BioMed Central will be the most significant development for disseminating the results of biomedical research in our lifetime. "

Sir Paul Nurse, Cancer Research UK

Your research papers will be:

- available free of charge to the entire biomedical community

- peer reviewed and published immediately upon acceptance

- cited in PubMed and archived on PubMed Central

- yours - you keep the copyright

Submit your manuscript here:

http://www.biomedcentral.com/info/publishing_adv.asp
BioMedcentral 